\title{
Long automated driving phase affects take-over performance
}

\author{
A. Bourrelly ${ }^{1 *}$, C. Jacobé de Naurois ${ }^{1}$, A. Zran ${ }^{2}$, F. Rampillon ${ }^{2}$, JL. Vercher ${ }^{1}$, C. Bourdin ${ }^{1}$ \\ ${ }^{1}$ Aix Marseille Univ, CNRS, ISM, Marseille, France \\ ${ }^{2}$ Groupe PSA, Centre Technique de Vélizy, Vélizy-Villacoublay, France \\ *aurore.bourrelly@hotmail.fr
}

\begin{abstract}
We investigated the impact of automated driving phases of different durations (long vs. short) on take-over performance and driver state. Thirty participants drove on a dynamic simulator under autonomous mode for three successive periods of automated driving: a short (10 $\mathrm{min})$, a long (1 hour) and another short (10 min) period, each ending with a take-over request. They performed a non-driving task, watching a film of their choice, throughout the autonomous phases. Driving performance - reaction time and quality - and driver drowsiness were assessed at each take-over. One hour of automated driving affected the driver's behaviour, leading to poorer take-over performance (longer reaction time and sharper avoidance manoeuvre) and increased drowsiness compared to a shorter autonomous period. Results also suggest that sequencing the autonomous phase in several short periods should improve the driver's take-over performance and help prevent drowsiness. Our findings sound a warning on the risks associated with long phases of automated driving. In particular, we provide evidence that series of short periods of automated driving are preferable to a long, continuous automated driving phase.
\end{abstract}

\section{Introduction}

With the emergence of new driving functions, Level 3 automation will enable users to be driven for lengthy periods under automation, at high speed and without worrying about road events $[1,2]$. For instance, the "highway chauffeur" function will handle the lateral and longitudinal controls of the vehicle for long periods and at high speed, without needing continuous driver supervision. Under appropriate conditions, the driver will be able to activate this function by pressing a button on the steering wheel before removing hands from the wheel and feet from the pedals and engaging at length in another activity. By handling the control of the vehicle, automation will allow drivers to focus on more pleasant activities like playing games or watching films instead of driving [3, 4]. However, when necessary (e.g., when the system's limits are reached due to sensor or actuator dysfunction, ambiguous environment observations, etc.), the current classification makes it clear that drivers must be able to regain control within a reasonable transition time, to deal with the driving event [5]. To this end, the system is designed to emit a takeover request (TOR) specifically intended to redirect the drivers' behaviour towards their prime activity.

Parallel to the numerous claimed or supposed benefits of automation (e.g., safety; economic, ecological, comfort; see [6]), a major issue arises here: that of a driver no longer engaged in the driving task safely resuming control. Handing over control to an autonomous system leaves the user "out-of-the-loop", which may induce deterioration of reaction time and inappropriate human behaviour in the event of a TOR [7, 8, 9, 10]. Previous research on automated driving demonstrated that drivers focusing on another task lose awareness of the situation, which impacts driving performance during take-over $[11,12$, 13, 14, 15, 16]. This reduced performance generally manifests itself in more abrupt braking reactions or avoidance manoeuvres and less stable trajectories $[11,17]$.
In addition, automated driving has also been shown to generate passive fatigue or mental underload, which can lead to increased driver drowsiness. Such impairments to the drivers' cognitive state can also be heightened by a monotonous non-driving-related task.

Car manufacturers, therefore, crucially need to better assess the relative safety of driver take-over after periods of automated driving. This will enable them to make the most appropriate recommendations for users wanting to take full advantage of this new technology.

The negative impact of automated driving, mainly linked to fatigue, appears to increase with time spent under automation $[4,15,18,19,20]$. A previous study found that drivers rated themselves as subjectively tired even after only $15 \mathrm{~min}$ of automated driving [21]. Objective measures of driver fatigue also confirmed an impairment of cognitive state during $20 \mathrm{~min}$ [22] or $42 \mathrm{~min}$ of automated driving [23] Moreover, findings by [18] suggested that under lengthy autonomous phases, drivers may not only be susceptible to fatigue but may also perform worse than manual drivers when faced with a critical event. However, most of these studies did not investigate whether such fatigue affected drivers' performance during take-over.

Taking-over control of automated vehicles has been dealt with extensively in the literature (see [24] for a review; [25] for modelling). Studies have investigated the impact on take-over performance of the time allowed for take-over [16, $26,27,28,29,30,31]$, of traffic density at take-over $[4,12$, 32 ], of the nature of the non-driving-related task performed by drivers $[11,14,33,34]$, or of how the TOR is issued [30, $35,36,37]$. However, most of these studies analysing the degradation of driving performance assessed participants exposed to short autonomous phases (from a few seconds to less than 5 minutes) prior to TOR [14, 27, 28, 36]. Paradoxically, few studies have addressed the impact of longer periods of un-interrupted automated driving (from 5 $\min$ to $30 \mathrm{~min}[18,22,38])$. For instance, [22] concluded that 20-minute autonomous phases induced slower reaction 
times, but no significant changes in accelerations and timeto-collision during take-over. Yet the original objective behind the function's design (to allow drivers to watch a movie, for example) means that one hour or more of automated driving would actually be more relevant to intended use than 30 minutes. To our knowledge, there is a lack of evidence regarding prolonged periods of exposure to automation. In addition, no study has so far dealt with the effect of successive TORs on the evolution of take-over performance, despite the fact that drivers may well be exposed to successive TORs in certain environmental contexts.

The aim of this study was therefore twofold. Firstly, we attempted to better address the real intended use of the function, by investigating the impact of a realistically long automated driving phase on take-over performance. We hypothesised that a lengthy (1 hour) autonomous phase would have stronger effects on driver's behaviour (in terms of both reaction time and quality) than a short phase (10 min), as compared to a reference manual driving phase (without automation). Secondly, since we expected the quality of take-over performance to decrease with longer automated driving, we investigated whether the risk associated with the long automated driving phase could be avoided by interspersing long and short periods of automated driving. Thus, our protocol included two short autonomous phases, one at either end of a longer autonomous phase. The impact of these long and short periods of automated driving was investigated under two traffic conditions: a) critical, where drivers had to avoid two cars that had crashed, and b) no traffic.

\section{Method}

\subsection{Participants}

Thirty daily drivers, 15 male, 15 female, aged between 35 and 55 (mean age $46 \pm 6$ years), took part in the experiment performed in a dynamic driving simulator. All were experienced drivers (more than $10000 \mathrm{~km} / \mathrm{year}$ and 15 years of driving experience), familiar with ADAS although unfamiliar with a dynamic simulator.

\subsection{Apparatus}

The experiment was conducted in the SHERPA ${ }^{2}$ dynamic driving simulator at PSA Research Centre. This simulator included a cell containing a half-cab where the driver sat, mounted on a hexapod and an X-Y motion platform (Fig. 1). The cell was fully equipped (2 front adjustable seats, seat belts, steering wheel, pedals, gearbox, rear-view and side-view digital mirrors) and had a sound restitution system composed of 6 loudspeakers and a subwoofer. The cell faced 3 flat screens placed side by side, providing a $120^{\circ}$ (horizontal) by $25^{\circ}$ (vertical) field of view. The rear view was displayed on three digital mirrors and dashboard information was provided by a 10 " $16 / 9$ LCD screen positioned behind the steering wheel. A $10^{\prime \prime}$ Microsoft Surface 3 installed on the central console of the simulator was used as a human-machine interface (HMI). The driving simulation was controlled by the $\mathrm{SCANeR}{ }^{\mathrm{TM}}$ studio software developed by Oktal@.

\subsection{Procedure}

After a briefing on the study and the setup, participants were instructed to drive in the right-hand lane of a two-lane highway at $110 \mathrm{~km} / \mathrm{h}$, in two driving sessions. They drove both sessions with an automatic gearbox. In the first session, participants first drove under manual mode for at least $10 \mathrm{~min}$ to familiarise them with the simulator. When they felt comfortable with the driving controls, a manual driving reference (MD) was recorded under two different traffic conditions (described below). After this, the participants familiarised themselves with the autonomous function by performing the following tasks: activating the function to engage the automation mode, removing hands and feet from the controls, launching a movie on the user display and finally regaining control in response to a TOR by placing hands and feet simultaneously on the driving controls. The system then switched from automation to a conventional manual mode as participants performed the take-over manoeuvre. Participants experimented with at least 4 TORs until they felt comfortable with the function, prior to the experimental sessions per se. The aim of this training was to reduce the variance resulting from learning effects previously found in take-over experiments [28]. This session lasted around $30 \mathrm{~min}$.

After a short break, the participants moved on to the second session. They spent about 90 min continuously in the simulator, alternating phases of automated driving and, after TOR, manual driving. At the beginning of this session, participants were asked to start driving and soon after to engage the autonomous function. There were three successive phases of automated driving: SHORT1 (10 min), LONG (1 hour), and SHORT2 (10 min), always presented in this order. Each automated phase ended with a request to regain control, yielding a total of 3 take-over requests (TORs). After each TOR, participants drove for about 2 minutes in manual mode before re-engaging the autonomous function. During the automated phases (SHORT1, LONG SHORT2), the drivers were engaged in an entertaining task: watching a film on the control screen behind the steering wheel. To ensure that the non-driving task was enjoyable, participants were allowed to choose from a list of recent films and to change films whenever they liked. The objective was to place the driver out-of-the-loop and to prevent him/her from obtaining information about the road event. At the time of the TOR, a visual-auditory signal, the film shut down (see Fig. 1).

Two traffic scenarios were used in this experiment, designed to reflect two scenarios classically encountered on a highway during a take-over situation. One involved an accident situation (CARS), where two cars that had crashed were immobile in the right lane and had to be avoided by moving to the left lane. The other was a no-car condition (NC), where there was no traffic. In the CARS condition, the surrounding vegetation masked the accident until the TOR, to avoid possible anticipation of the event if participants glanced at the road [26]. The experiment was designed so that the road was clear of other vehicles (except the two immobile cars) at the time of the TOR, to ensure standardised take-over. A traffic flow remained displayed on the other side of the highway. 


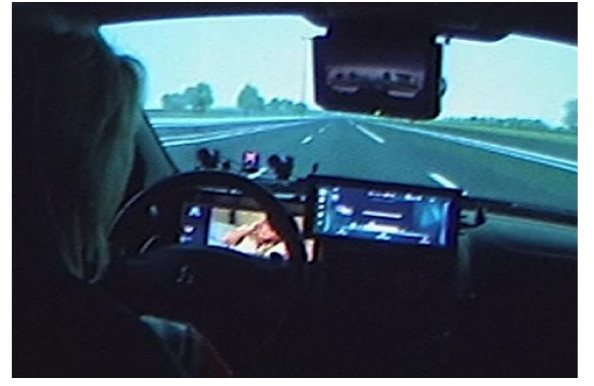

Fig.1. Wide-angle shot of the simulator from the driver's seat

Traffic scenarios were counterbalanced across participants in four groups, with an overlapped design for autonomous phase duration (SHORT1-LONG-SHORT2) and traffic (CARS-NC). The distribution of the traffic conditions for the three automated driving durations is given in Table 1. Whatever the traffic condition, the drivers had $10 \mathrm{~s}$ after the TOR to regain control. At the time of the TOR, the accident was $305 \mathrm{~m}$ ahead of the participant's car.

Table 1 Distribution of the traffic conditions experienced in the three automated driving durations for each of the four groups of participants

\begin{tabular}{l|ccc}
\hline Group & SHORT1 & LONG & SHORT2 \\
\hline Group 1 & NC & NC & CARS \\
Group 2 & NC & CARS & CARS \\
Group 3 & CARS & CARS & NC \\
Group 4 & CARS & NC & NC \\
\hline
\end{tabular}

Performance data (reaction times and car trajectories) were extracted directly from the simulator and recorded for analysis. In parallel, subjective measures of driver state and perceived performance of the manoeuvre were collected. Driver's drowsiness was verbally assessed after each TOR using a five-level Likert scale ranging from 1 (alert / wide awake), 2 (a little drowsy), 3 (drowsy), 4 (very drowsy), to 5 (extremely drowsy). Scores were given just after the autonomous mode was re-engaged and before the film was re-started. Although expressed a few minutes later, the scores had to specifically represent the level of drowsiness felt at the time of the TOR. Participants also gave their opinion on other aspects: the perceived effectiveness of the TOR, the ease-of-use of the function, the adequacy of the $10 \mathrm{~s}$ given to intervene, and the perceived safety of the takeover manoeuvre. Participants' subjective ratings were recorded on a five-level Likert Scale ranging from 1 (not at all satisfied), 2 (fairly dissatisfied), 3 (partially satisfied), 4 (quite satisfied), to 5 (completely satisfied). Trust in the automated system was also assessed before and after they performed the experiment, using the same five-level Likert Scale. There was a short debriefing session immediately after the experiment to assess participant perception. Apart from these subjective measures, the experimenters never talked to the driver during the automated driving phases.

\subsection{Dependent variable descriptions}

Take-over performance (reaction time and quality) was assessed during the three following phases:

Transition phase included the time from the TOR being issued until the driver placed hands on the steering wheel and placed feet on the pedals (brake or accelerator), and when the function actually disabled.

Avoidance manoeuvre, (measured for the CARS condition only) described the vehicle dynamics when bypassing the two cars involved in the accident. It included the time from the TOR being issued until the drivers changed lane and returned to the righthand lane; the distance and time to collision (DTC and TTC, respectively); lateral deviation at the place of the accident; and maximum lateral speeds when bypassing and returning. According to Gold et al. (2015) [27], lower accelerations and higher TTC should represent a safer handling of the situation and therefore better take-over quality. The distance and time required to return to the righthand lane relative to the place of accident (DTR and TTR, respectively) were also assessed, as an indicator marking the end of the avoidance manoeuvre.

Quality of control highlighted differences between the autonomous conditions and the MD reference. Individual standard deviations of lateral deviation, steering angle, longitudinal and lateral speeds (averaged by $60 \mathrm{~m}$ increments) for the long and short autonomous phase conditions were compared to the manual driving condition. These analyses indicated the point in the take-over manoeuvre when participants' driving behaviour once again became comparable to that of MD.

\subsection{Statistical analyses}

For all the following statistical analyses, data normality was first assessed using the D'Agostino-Pearson $\mathrm{K}^{2}$ test. If the null hypothesis of the normality test was rejected, an equivalent non-parametric permutation procedure was applied [39]. The $\alpha$-level of significance was set at 0.05 for all statistical tests.

Regarding the transition phase, a mixed ANOVA with two factors was run to examine the impact of LONG vs. SHORT automated driving phases (within-subjects factor) under both traffic conditions (between-subjects factor) Within-subjects ANOVAs were run to examine the impact of driving condition (LONG, SHORT and MD) on vehicle dynamics during the avoidance manoeuvre. Note that SHORT indicates either SHORT 1 or SHORT2 according to the traffic condition across the four groups of participants (see Table 1). Pairwise comparisons with Bonferroni corrections were used for post-hoc analyses. To assess the quality of control, within-subjects $t$-tests with a statistical parametric mapping method [40] were used to compare each automated driving profile to MD reference.

To test our second hypothesis on the take-over performance variables, SHORT1 and SHORT2 conditions were compared. Since there was no intra-individual variability (only one take-over per condition), betweensubjects $t$-tests were used instead of ANOVAs for these analyses. 


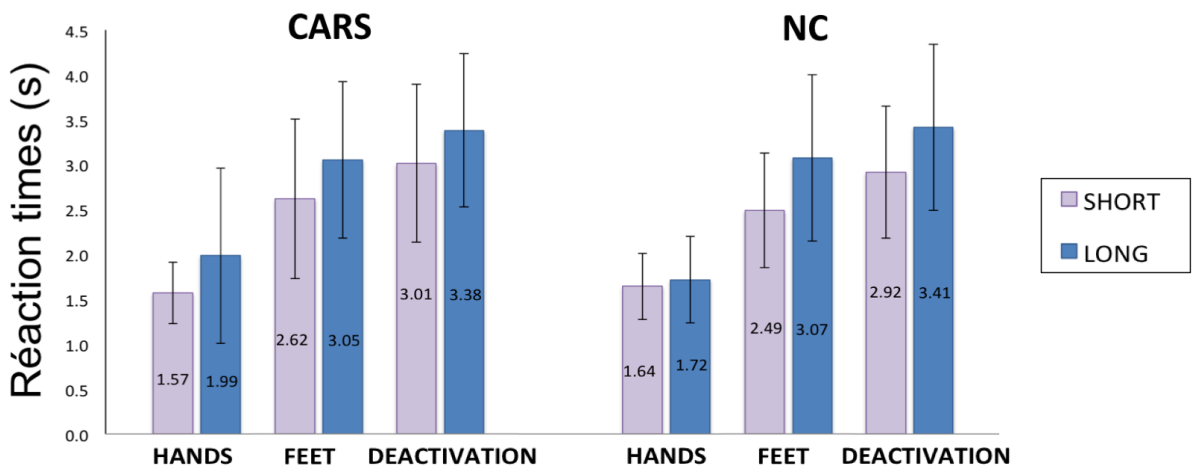

Fig.2. Mean of reaction times in take-over phase for SHORT and LONG automatic driving conditions in both CARS and NC traffic conditions. Errors bars represent standard deviation $( \pm S D)$. Statistics are reported in Table 2. Reaction time: "time required to perform a physical act (braking, steering or pressing a button) in response to some external event" [41]

Regarding driver state, within-subjects ANOVAs were used to assess the impact of the automated driving conditions on self-perceived drowsiness state and on the other subjective scores. Pairwise comparisons with Bonferroni corrections were used for post-hoc analyses.

\section{Results}

All participants completed the experiment. Overall, they found the study enjoyable and the films entertaining. None suffered from simulator sickness.

\subsection{Performance data and take-over quality}

Transition phase: All drivers intervened within the given $10 \mathrm{~s} .74 \%$ of the participants regained control in the first $4 \mathrm{~s}$ following the TOR, regardless of automated driving duration. ANOVA results showed a significant effect of autonomous phase duration for each action (see Table 2). As illustrated in Fig. 2, hands on the steering wheel, first action on the pedals and function deactivation occurred about $0.5 \mathrm{~s}$ later in the LONG condition, whatever the traffic condition. There was no effect of traffic condition and no effect of interaction.

Table 2 ANOVA main effects of automated driving duration (SHORT vs. LONG) and traffic (CARS vs. $\mathrm{NC}$ ) during take-over phase

\begin{tabular}{l|lll}
\hline Transition phase & $\boldsymbol{F ( 1 , 2 8 )}$ & $\boldsymbol{p}$ & $\boldsymbol{\eta}_{\boldsymbol{p}}{ }^{2}$ \\
\hline Hands on steering wheel & & & \\
Duration & 3.75 & 0.050 & 0.12 \\
Traffic & 0.30 & 0.624 & 0.01 \\
Duration X Traffic & 1.88 & 0.174 & 0.06 \\
Feet on the pedal & & & \\
Duration & 13.23 & 0.001 & 0.32 \\
Traffic & 0.04 & 0.846 & 0.00 \\
Duration X Traffic & 0.30 & 0.589 & 0.01 \\
Deactivation & & & \\
Duration & 7.78 & 0.009 & 0.22 \\
Traffic & 0.01 & 0.907 & 0.00 \\
Duration X Traffic & 0.18 & 0.678 & 0.01 \\
\hline \multicolumn{4}{|l}{}
\end{tabular}

Avoidance manoeuvre: The ANOVAs showed a significant effect of automated driving condition on lane change, return to righthand lane, DTC and TTC, and maximum lateral speeds when bypassing and returning (see Table 3). Posthoc analyses showed that after the LONG autonomous phase, lane change occurred about $0.8 \mathrm{~s}$ later than in the SHORT conditions averaged out $(\mathrm{p}<.001)$ and $1 \mathrm{~s}$ later than in the MD condition. TTC and DTC were de facto shorter $(\mathrm{p}<.001)$ after the LONG autonomous phase. TTC was about $0.7 \mathrm{~s}$ and $1.6 \mathrm{~s}$ shorter in the LONG condition than in the averaged SHORT conditions and MD, respectively. Lateral speed was also higher in the LONG condition when bypassing the accident and returning to the righthand lane ( $\mathrm{p}<.05)$. For example, maximum lateral speed when bypassing the accident was about $0.14 \mathrm{~m} . \mathrm{s}^{-1}$ and $0.45 \mathrm{~m} . \mathrm{s}^{-1}$ higher in the LONG condition than in the SHORT conditions and MD, respectively, while maximum lateral speed when returning to the righthand lane was about 0.17 $\mathrm{m} . \mathrm{s}^{-1}$ and $0.16 \mathrm{~m} \cdot \mathrm{s}^{-1}$ higher in the LONG condition than in the SHORT conditions and MD, respectively. Nevertheless, no significant difference between conditions was found in lateral deviation ( $\mathrm{p}=.30$; about $1.8 \mathrm{~m}$ from the central line) Similarly, no difference was found in DTR $(\mathrm{p}=.72)$ and TTR $(p=.12)$. These results suggest that driving behaviour stabilised after bypassing the accident (for example, drivers returned to the righthand lane on average $85 \mathrm{~m}$ and $3.7 \mathrm{~s}$ after bypassing the accident). Mean trajectory profiles of driving behaviour for all conditions are illustrated in Fig 3 .

Quality of control: Variability in the control of the vehicle (SD of lateral deviation, steering angle, longitudinal and lateral speeds) was higher after the LONG autonomous phase compared to $\mathrm{MD}$, but mainly before bypassing the accident (300 $\mathrm{m}$ after the TOR). Compared to MD, the SD of lateral deviation in the LONG condition was lower at $121-180 \mathrm{~m}(\mathrm{p}=.048)$ and higher at $241-300 \mathrm{~m}(\mathrm{p}=.046)$ after the TOR. The SD of lateral speed was higher for all automated driving conditions compared to $\mathrm{MD}$ at 121 $180 \mathrm{~m}(\mathrm{p}<.05)$, and for the LONG condition alone at 241$300 \mathrm{~m}$, after the TOR $(\mathrm{p}=.05)$. The SD of steering angle was higher only in the SHORT2 condition compared to MD at 61-120 $\mathrm{m}$ after the TOR $(\mathrm{p}=.049)$. 
Table 3 Mean of driving performance $( \pm \mathrm{SD})$ and ANOVA main effects during the avoidance manoeuvre.

${ }^{\mathrm{M}}$ indicates significant difference from MD condition. ${ }^{\mathrm{s}}$ indicates significant difference from SHORT condition.

\begin{tabular}{c|cccccc}
\hline Avoidance Manoeuvre & MD & SHORT & LONG & $\boldsymbol{F ~ ( 2 , 2 8 )}$ & $\boldsymbol{p}$ & $\boldsymbol{\eta}_{\boldsymbol{p}}{ }^{2}$ \\
\hline Lane Change (s) & 7.05 & 7.29 & $8.06^{\mathrm{M} . \mathrm{S}}$ & 5.87 & 0.007 & 0.30 \\
& $(1.16)$ & $(0.90)$ & $(0.75)$ & & & \\
Return (s) & 16.27 & $14.60^{\mathrm{M}}$ & $14.07 \mathrm{M}$ & 7.42 & 0.008 & 0.35 \\
& $(3.11)$ & $(2.38)$ & $(1.97)$ & & & \\
DTC (m) & 100.12 & 87.53 & $66.30^{\mathrm{M} . \mathrm{s}}$ & 9.01 & 0.001 & 0.39 \\
& $(29.01)$ & $(25.72)$ & $(20.84)$ & & & \\
TTC (s) & 4.08 & $3.22^{\mathrm{M}}$ & $2.49^{\mathrm{M} . \mathrm{S}}$ & 13.38 & $<0.001$ & 0.49 \\
& $(1.16)$ & $(0.92)$ & $(0.79)$ & & & \\
Lateral deviation & 1.84 & 1.82 & 1.72 & 1.24 & 0.304 & 0.08 \\
& $(0.17)$ & $(0.25)$ & $(0.29)$ & & & \\
Maximum Lateral speed (bypassing) & 0.84 & $1.15^{\mathrm{M}}$ & $1.29^{\mathrm{M}}$ & 15.16 & 0.002 & 0.52 \\
Maximum Lateral speed (returning) & $(0.19)$ & $(0.29)$ & $(0.39)$ & & & \\
& 1.06 & 1.07 & 1.23 & 3.07 & 0.062 & 0.18 \\
& $(0.33)$ & $(0.26)$ & $(0.33)$ & & & \\
\hline
\end{tabular}

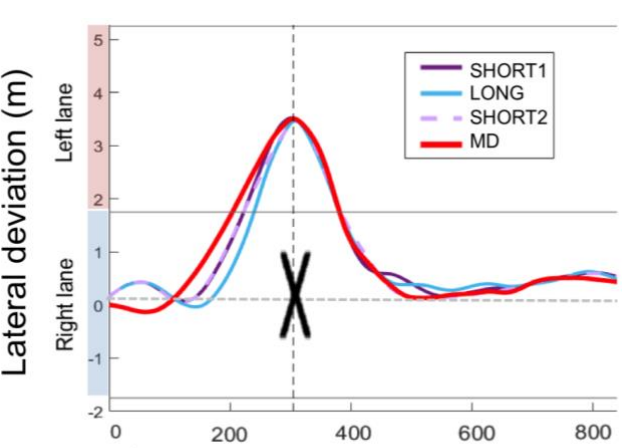

(j)) Distance from the TOR (m)

Fig.3. Mean trajectory profiles of the avoidance manoeuvre in the CARS condition for all driving conditions (SHORT1, LONG, SHORT2 and MD reference). The black cross marks the accident. The horizontal dashed line represents the middle of the right lane.

Regarding our second hypothesis, between-subjects t-tests did not show any significant differences between SHORT1 and SHORT2 in any variables.

\subsection{Driver state}

Drivers reported becoming very drowsy after a long period of automated driving (see Fig. 4). Forty per cent of the subjective drowsiness scores switched from a little and partially drowsy (score ranging from 1 to 3 ) to very and extremely drowsy (score about 4 and 5) in the LONG condition. As confirmed by experimenter observation, six of the 30 participants self-reported temporarily sleeping during the long autonomous phase Consistent with our main hypothesis, results highlighted a significant effect on drowsiness of the long autonomous phase compared to the two short phases $(p<.001)$. Importantly, drowsiness scores did not differ significantly between the two SHORT conditions $(\mathrm{p}=.182)$. Figure 4 shows the distribution of participants' drowsiness scores for the three durations of autonomous phase.

\subsection{Perceived performance}

Participants reported being generally satisfied with the effectiveness of the TOR signal and the ease-of-use of the function. They also reported being generally satisfied with the $10 \mathrm{~s}$ allowed for take-over and with the perceived safety of the take-over manoeuvre (mean satisfaction scores for all items $>4 / 5$; see Table 4). As to their trust in the automation, participants reported being generally confident of the system's reliability. Scores increased after trying out the system (mean score before: $\mathrm{M}=3.77, \mathrm{SD}=1.01$; after: $\mathrm{M}=4.00, \mathrm{SD}=0.75)$, reaching significance ( $\mathrm{p}<.001)$.

Higher scores were awarded in the SHORT2 condition than in the LONG and SHORT1 conditions for the effectiveness of the TOR signal $(p<.05)$, the adequacy of the $10 \mathrm{~s}$ allowed for take-over $(\mathrm{p}<.01)$, and the perceived safety of the take-over manoeuvre $(\mathrm{p}<.01)$. Although satisfaction scores on the function's ease-of-use were generally high, participants reported being slightly less satisfied in the LONG condition than in the SHORT1 $(\mathrm{p}=.05)$ and SHORT2 ( $\mathrm{p}=.044)$ conditions.

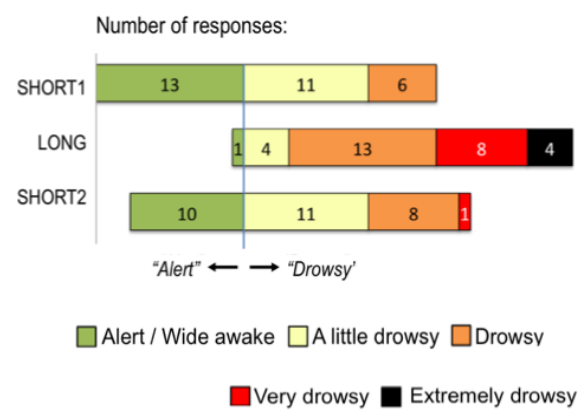

Fig.4. Distribution of the drowsiness scores as rated by participants for the three automated driving conditions (SHORT1, LONG, SHORT2). Bars are aligned relative to the "Alert" state. 
Table 4 Mean of satisfaction scores, (SD), and ANOVA main effects of automated driving duration on factors in perceived performance. ${ }^{\mathrm{S} 2}$ indicates a significant difference from SHORT2.

\begin{tabular}{c|cccccc}
\hline Perceived performance & SHORT1 & LONG & SHORT2 & $\boldsymbol{F ~ ( 2 , 5 8 )}$ & $\boldsymbol{p}$ & $\boldsymbol{\eta}_{\boldsymbol{p}}{ }^{\mathbf{2}}$ \\
\hline Effectiveness & $4.43^{\mathrm{S} 2}$ & $4.40^{\mathrm{S} 2}$ & 4.70 & 3.43 & 0.032 & 0.11 \\
of the signal & $(0.73)$ & $(0.72)$ & $(0.60)$ & & & \\
Ease-of-use & 4.63 & 4.50 & 4.73 & 2.01 & 0.112 & 0.06 \\
of the function & $(0.56)$ & $(0.68)$ & $(0.52)$ & & & \\
Adequacy & $3.87^{\mathrm{S} 2}$ & $4.10^{\mathrm{S} 2}$ & 4.50 & 6.26 & 0.004 & 0.18 \\
of time allowed & $(0.90)$ & $(0.96)$ & $(0.73)$ & & & \\
Safety & 4.07 & 4.07 & 4.40 & 3.26 & 0.052 & 0.10 \\
of the manoeuvre & $(0.69)$ & $(0.74)$ & $(0.72)$ & & & \\
\hline
\end{tabular}

\section{Discussion}

The two objectives of this study were 1) to assess how the length of automated driving phases (long vs. shor periods) might impact take-over performance and 2) to assess whether interspersing long and short periods of automated driving might have a beneficial effect on the driver's state, and thus on take-over performance. Long (1 hour) and short $(10 \mathrm{~min})$ periods under autonomous mode were compared for their effect on the take-over manoeuvre. To assess quality of control, the different durations of autonomous phase were also compared to a reference manual driving condition. Finally, performance data for the two short autonomous phase conditions were compared, to determine whether there was an advantage to sequencing several shorter autonomous periods.

\subsection{Impact of duration of automated driving}

Our results clearly show that the duration of automated driving affects take-over performance and quality. One hour of automated driving resulted here in drivers taking an average of some $0.5 \mathrm{~s}$ longer to begin the series of actions involved in changing lanes when bypassing an accident. This increased reaction time, observed across driving actions, suggests a general shift in the execution of the driving motor sequence. A possible cause may be deterioration of the drivers' cognitive state due to a long period of automated driving prior to TOR [18, 42]. As previously found by [43], longer reaction times may indicate a decrease in vigilance level and an increase in fatigue. This is supported by the participants' reports that their drowsiness increased with the time spent under autonomous mode.

When it comes to the avoidance manoeuvre and quality of control, marked differences were found between the conditions. For example, drivers changed lane later to bypass the accident under both long and short automated driving conditions compared to the manual driving reference. This resulted in a reduced longitudinal safety margin to cope with the situation before bypassing the accident. In addition, lateral speed during bypassing and return was higher after a period under autonomous mode, i.e., drivers performed a sharper avoidance manoeuvre. Importantly, these results were even more marked after one hour of automated driving, pointing to increasing criticality with time spent under autonomous mode. Regarding the quality of control,variability in take-over behaviour was mainly observed in the first $300 \mathrm{~m}$, suggesting that there was greatest instability before the accident was reached. While bypassing the accident, drivers showed no difference in lateral deviation between conditions, still managing a comfortable lateral safety margin (about $1.8 \mathrm{~m}$ from the central lane). After they passed the accident, there was no difference in the time and distance drivers took to return to the righthand lane, suggesting that driving behaviour stabilised very soon after they reached the accident.

Overall, the present results are consistent with the literature reporting a deterioration of take-over performance after a period spent under autonomous mode as compared to manual driving $[11,14]$. However our main findings, which show an autonomous phase duration effect, contrast with previous work by [22], who failed to observe any difference in take-over performance between 5- and 20-minute periods of automated driving. The authors explained their result as potentially due to their autonomous phases, being too short to induce any obvious deterioration related to fatigue or hypo-vigilance. They also provided another explanation: the fatigue induced by a substantial training session might have been sufficient to cause deterioration of take-over performance after only five minutes of automated driving.

In our study, there were significant differences between the effects of short 10-min and long 1-hour autonomous periods. As shown by the high level of reported drowsiness, watching a film over a long period provided stronger passive fatigue and cognitive underload due to the monotony of the task. Longer reaction times and greater variability in the quality of driving control are indicative of drivers being out-of-the-loop [13]. This is, to our knowledge, the first study showing a detrimental effect of a long automated driving phase, not only on reaction times but also on the quality of take-over.

\subsection{Beneficial effect of shorter automation periods}

To assess whether there is any advantage to sequencing automation in several shorter periods, we compared performance data from the two short duration conditions. Since deterioration in take-over performance has been considered to be related mainly to the driver's cognitive underload [15, 44, 45, 46, 47], we expected an improvement 
in driver behaviour when shorter automation periods surrounded the long one. In fact, our shorter automation periods meant more frequent TORs, forcing drivers to interrupt the non-driving-related task and redirect their attention to the road. Thus, allowing more frequent alternation between automated and manual driving could be expected to help drivers maintain a good level of vigilance over time (i.e., to limit the impact of being out-of-the-loop), thereby enhancing safety during take-over.

Consistent with this assumption, there was no difference between the two short conditions in performance variables. Reaction times (i.e., hands on the steering wheel, feet on the pedals and function deactivation) were comparable. Measurements throughout the avoidance manoeuvre were similar, with vehicle dynamics describing overlapping profiles.

Most importantly, in terms of drivers' drowsiness, scores did not differ significantly between the two short conditions. As previously described, drowsiness was rated higher after the LONG autonomous phase, supporting an increased level of fatigue. However, despite the fact that more than one hour of automated driving separated the two short periods, participants reported a fairly similar level of drowsiness in the SHORT2 condition as in the SHORT1. Taking-over control at the end of the long autonomous period appears to have erased the impact of the accumulated passive fatigue, and a beneficial effect on the driver's state was retained even after a further 10-minute autonomous period. This is particularly important because, to our knowledge, it provides the first evidence that sequencing long and short periods of automated driving can enhance driving safety during take-over.

\subsection{Perceived performance \\ \& Recommendations for use}

Driving in autonomous mode was generally viewed positively by the participants. Scores were high on all items, regardless of automated driving duration. This confirms that the design of the function, including the nature of the TOR signal, appropriately addressed the situations encountered. Only a slight impact of the long autonomous period was found on perceived ease-of-use of the function, expressed by participants as a consequence of the time pressure they felt when faced with bypassing the accident. On the other hand, the higher satisfaction scores in the SHORT2 condition are consistent with increased trust in the autonomous function after experience with the system, in accordance with previous observations in the literature [22, 48].

Overall, these good satisfaction scores are consistent with the objective performances measured in this experiment. All participants were able to take control in 3.1 $\mathrm{s} \pm 0.8 \mathrm{~s}$ (response time consistent with previous findings by Melcher et al, 2016 [29] : about $3.5 \mathrm{~s}$ for a similar allowance of time) and cope safely with the driving situation. For example, even when participants changed lanes later they managed a reasonable lateral safety margin whatever the driving condition. It is noteworthy that the present study only dealt with simple take-over situations (no surrounding traffic except the two crashed cars, a comfortable time allowance and no sudden unexpected events). This is illustrated by the lack of difference in results between the two traffic conditions (CARS vs. NC). Caution should be used in extending its implications regarding safety or risklevel to more complex situations.

Taken together, our findings sound a note of warning on the risk associated with long periods of automated driving. It is therefore vital to seek ways of making the use of automation functions safer. A recent study revealed that drivers are unable to stay alert during extended periods of automated driving without non-driving-related tasks [20]. The present results suggest that even with non-drivingrelated tasks, especially when these are prone to induce mental underload or drowsiness, there could be serious risk in more complex take-over situations. Preventive solutions could include using more diverse non-driving tasks to break the monotony $[15,34,46,47,49,50]$, or providing a road awareness recall when the out-of-the-loop state (or drowsiness) is detected or predicted by the system [51]. These solutions could feature as recommendations for use of the function, enabling drivers to enjoy the benefits of long periods under autonomous mode while maintaining good awareness of the road situation. The present work suggests that varied tasks and road awareness recall methods are worth investigating.

\section{Conclusion}

Our findings demonstrate that one hour of automated driving affects the driver's behaviour, leading to a decline in take-over performance and increased drowsiness. Although the true level of risk remains to be quantified before we can fully conclude on driver safety, this study sounds a warning note about the risks associated with a long period of automated driving, especially when take-over involves an unexpected event. Crucially, our findings suggest that more frequent TORs might improve driver behaviour during takeover. To allow drivers to safely take advantage of long periods of automated driving, it would be worth seeking solutions that can feature as recommendations for use of the autonomous function. It would also be particularly valuable to investigate whether more complex scenarios involving more traffic, other types of secondary task and longer periods of automated driving might provide broader insights into the problem.

\section{Acknowledgments}

Aurore Bourrelly was supported by a post-doctoral research fellowship from Groupe PSA via the OpenLab agreement with Aix-Marseille University and CNRS entitled "Automotive Motion Lab". The authors thank Gilles Houzé of PSA for his valuable help with SCANeR® Software Developments, Celine Taccory D for funding, Marjorie Sweetko for English language editing and Dr Cédric Morio for his advice on statistical processing.

\section{References}

[1] Gasser, T.M. and Westhoff, D.: 'BASt-study: definitions of automation and legal issues in Germany', Road Vehicle Automation Workshop, Irvine, CA, United States, July 2012

[2] 'SAE Taxonomy and Definitions for Terms Related to On-Road Motor Vehicle Automated Driving Systems', 
http://standards.sae.org/j3016 201401/, accessed: 25 October 2016

[3] Carsten, O., Lai, F.C.H., Barnard, et al.: 'Control task substitution in semi-automated driving: Does it matter what aspects are automated?', Hum. Factors, 2012, 54, (5), pp. 747-761

[4] Jamson, A.H., Merat, N., Carsten, O.M., et al.: 2013. 'Behavioural changes in drivers experiencing highlyautomated vehicle control in varying traffic conditions', Transp. Res. Part C, 2013, 30, pp. 116-125

[5] 'NHTSA U.S. Department of Transportation Releases Policy on Automated Vehicle Development', http://www.nhtsa.gov/About-NHTSA/Press-Releases/U.S.Department-of-Transportation-Releases-Policy-onAutomated-Vehicle-Development, accessed: 25 October 2016

[6] Stanton, N.A., Young, M.S.: 'Vehicle automation and driving performance', Ergonomics, 1998, 41, (7), pp. 1014 1028

[7] Bainbridge, L.: 'Ironies of automation', Automatica, 1983, 19, (6), pp. 775-779

[8] Brookhuis, K.A., de Waard, D., Janssen, W.H.: 'Behavioural impacts of Advanced Driver AssistanceSystems-an overview', Eur J Transp Infrast, 2001, 1, (3), pp. 245-253

[9] Endsley, M.R.: 'Toward a theory of situation awareness in dynamic systems', Hum. Factors, 1995, 37, (1), pp. 32-64

[10] Wiener, E.L., Curry, R.E.: 'Automation in the Cockpit: Some Generalizations', Proc. Hum. Factors Erg. Soc. Annu. Meet., 1980, 24, (1), pp. 38

[11] Bueno, M., Dogan, E., Hadj Selem, F., et al.: 'How different mental workload levels affect the take-over control after automated driving', IEEE 19th International Conference on Intelligent Transportation Systems (ITSC), Rio de Janeiro, Brazil, November 2016, pp. 2040-2045.

[12] Gold, C., Körber, M., Lechner, D., Bengler, K.: 'Taking over control from highly automated vehicles in complex traffic situations - The role of traffic density', Hum. Factors, 2016, 58, (4), pp. 642-652

[13] Louw, T., Merat, N.: 'Are you in the loop? Using gaze dispersion to understand driver visual attention during vehicle automation', Transp. Res. C-Emer., 2017, 76, pp. $35-50$

[14] Merat, N., Jamson, A.H., Lai, F. C., Carsten, O.: 'Highly automated driving, secondary task performance, and driver state', Hum. Factors, 2012, 54, (5), pp. 762-771

[15] Naujoks, F., Höfling, S. Purucker, C., Zeeb, K.: 'From partial and high automation to manual driving: Relationship between non-driving related tasks, drowsiness and take-over performance', Accid. Anal. Prev., 2018, 121, pp. 28-42

[16] Zeeb, K., Buchner, A., Schrauf, M.: 'What determines the take-over time? An integrated model approach of driver take-over after automated driving', Accident Anal. Prev., 2015,78 , pp. 212-221

[17] Merat, N., Jamson, A. H., Lai, F. C., et al.: 'Transition to manual: Driver behaviour when resuming control from a highly automated vehicle', Transp. Res. F-Traf., 2014, 27, (B), pp. 274-282

[18] Neubauer, C., Matthews, G., Langheim, L., Saxby, D.: 'Fatigue and voluntary utilization of automation in simulated driving', Hum. Factors, 2012, 54, (5), pp. 734-746

[19] Saxby, D.J., Matthews, G., Hitchcock, et al.: 'Effects of active and passive fatigue on performance using a driving simulator', Proc. Hum. Factors Ergon. Soc. Annu. Meet., 2008, 52, (21), pp. 1751-1755

[20] Vogelpohl, T., Kühn M., Hummel, T., Vollrath, M. 'Asleep at the automated wheel-Sleepiness and fatigue during highly automated driving', Accid. Anal. Prev., 2018, In press.

[21] Gonçalves, J., Happee, R., Bengler, K.: 'Drowsiness in conditional automation: proneness, diagnosis and driving performance effects', IEEE 19th International Conference on Intelligent Transportation Systems (ITSC), Rio de Janeiro, Brazil, November 2016, pp. 873-878.

[22] Feldhutter, A., Gold, C., Schneider, S., Bengler, K.: 'How the Duration of Automated Driving Influences TakeOver Performance and Gaze Behavior', in Schlick, C. et al. (Eds.): 'Advances in Ergonomic Design of Systems, Products and Processes' (Springer, Berlin, Heidelberg, 2017), pp. 309-318

[23] Körber, M., Cingel, A., Zimmermann, M., Bengler, K.: 'Vigilance Decrement and Passive Fatigue Caused by Monotony in Automated Driving', Procedia Manuf., 2015, 3 , pp. 2403-2409

[24] Eriksson, A., Stanton, N.A.: 'Take-over time in highly automated vehicles: non- critical transitions to and from manual control', Hum. Factors, 2017, 59, (4), pp. 689-705

[25] Gold, C., Happee, R., Bengler. K.: 'Modeling take-over performance in level 3 conditionally automated vehicles', Accid. Anal. Prev., 2018, 116, pp. 3-13

[26] Gold, C., Damböck, D., Lorenz, L., Bengler, K.: "“Take over!" How long does it take to get the driver back into the loop?' Proc. Hum. Factors Ergon. Soc. Annu. Meet., 2013, 57, (1), pp. 1938-1942

[27] Gold, C., Berisha, I., Bengler, K.: 'Utilization of Drivetime - Performing Non-Driving Related Tasks While Driving Highly Automated', Proc. Hum. Factors Ergon. Soc. Annu. Meet., 2015, 59, (1), pp. 1666-1670 
[28] Gold, C., Bengler, K.: 'Taking over control from highly autonomous vehicles', in: Ahram, T., Jang, R.L. (Eds.), 'Proceedings of the 5th AHFE Conference Vol. 7', Kraków, Poland, July 2014, pp. 3662-3667

[29] Melcher, V., Rauh, S., Diederichs, F., et al.: 'Take-over requests for automated driving', Procedia Manuf., 2015, 3, pp. $2867-2873$

[30] Walch, M., Lange, K., Baumann, M., Weber, M.: 'Autonomous Driving: Investigating the Feasibility of CarDriver Handover Assistance', AutomotiveUI '15,

Nottingham, United Kingdom, September 2015, pp. 11-18

[31] Zeeb, K., Buchner, A., Schrauf, M.: 'Is take-over time all that matters? The impact of visual-cognitive load on driver take-over quality after conditionally automated driving', Accid. Anal. Prev., 2016, 92, pp. 230-239

[32] Radlmayr, J., Gold, C., Lorenz, L., et al.: 'How traffic situations and non-driving related tasks affect the take-over quality in highly automated driving', Proc. Hum. Factors Ergon. Soc. Annu. Meeting, 2014, 58, (1), pp. 2063-2067

[33] Llaneras R.E., Salinger, J., Green, C.A.: 'Human factors issues associated with limited ability autonomous driving systems: drivers' allocation of visual attention to the forward roadway', Driving Assessment 2013, Bolton Landing, NY, United States, June 2013, pp. 92-98

[34] Naujoks, F., Befelein, D., Wiedemann, K., Neukum, A.: 'A review of Non-driving- related tasks used in studies on automated driving', in: Stanton N. (Eds.), Advances in Human Aspects of Transportation, (AHFE 2017), Los Angeles, CA, United State, June 2017, pp. 525-537

[35] Beller, J., Heesen, M., Vollrath, M.: 'Improving the driver-automation interaction an approach using automation uncertainty', Hum. Factors, 2013, 55, (6), pp. 1130-1141

[36] Louw, T., Merat, N., Jamson, A.H.: 'Engaging With Highly Automated Driving: To Be Or Not To Be In The Loop?', Driving Assessment 2015, Salt Lake City, UT, United States, June 2015, pp. 189-195

[37] Naujoks, F., Mai, C., Neukum, A.: 'The effect of urgency of take-Over requests during highly automated driving under distraction conditions', in: Ahram, T., Jang, R.L. (Eds.), 'Proceedings of the 5th AHFE Conference Vol. 7', Kraków Poland, July 2014, pp. 2099-2106

[38] Bueno, M., Dogan, E., Hadj Selem, F., et al.: 'The effects of mental workload and duration of automated driving on driver behaviour', Proc. Int. Conf. Driver Distraction and Inattention, Paris, France, March 2017, pp. 32-33

[39] Nichols, T.E., Holmes, A.P.: 'Nonparametric permutation tests for functional neuroimaging: a primer with examples', Hum. Brain Mapp., 2002, 15, (1), pp. 1-25
[40] 'SPM1D 0.4' www.spm1d.org, accessed 4 October 2018

[41] Wintersberger, P., Green, P,. Riener, A.: 'Am I driving or are you or are we both? A taxonomy for handover and bandback in automated driving', Driving Assessment 2017, June 2017, Manchester Village, VT, pp. 333-339.

[42] Saxby, D.J., Matthews, G., Warm, J.S., et al.: 'Active and passive fatigue in simulated driving: discriminating styles of workload regulation and their safety impacts'. J Exp. Psychol. Appl., 2013, 19, (4), pp. 287-300

[43] Graw, P., Kräuchi, K., Knoblauch, V., et al.: 'Circadian and wake- dependent modulation of fastest and slowest reaction times during the psychomotor vigilance task'. Physiol. Behav., 2004, 80, (5), pp. 695-701

[44] Bourrelly, A., Jacobé de Naurois, C., Zran, A., et al.: 'Impact of a long autonomous driving phase on take-over performance'. Proc. Int. Conf. Driver Distraction and Inattention, Gothenburg, Sweden, October 2018, pp. 161164

[45] Bourrelly, A., Jacobé de Naurois, C., Zran, A., et al.: 'Gaze behavior during take-over after a long period of autonomous driving: A pilot study'. Proc. Int. Conf. Driving Simulation Conference Europe VR, Antibes, France, September 2018

[46] Jarosch, O., Kuhnt, M., Paradies, S., Bengler, K.: 'It's out of our hands now! Effects of non-driving related tasks during highly automated driving on drivers' fatigue'. Driving Assessment 2017, June 2017, Manchester Village, VT, pp. 319-325

[47] Marberger, C., Mielenz, H., Naujoks, F., et al.: 'Understanding and Applying the Concept of "Driver Availability" in Automated Driving'. in: Stanton N. (Eds.), Advances in Human Aspects of Transportation, (AHFE 2017), Los Angeles, CA, United State, June 2017, pp. 595-605

[48] Gold, C., Körber, M., Hohenberger, C., et al.: 'Trust in automation - Before and after the experience of take-over scenarios in a highly automated vehicle', Procedia Manuf., 2015, 3, pp. 3025-3032

[49] Naujoks, F., Wiedemann, K., Schömig, N.: 'The Importance of Interruption Management for Usefulness and Acceptance of Automated Driving'. AutomotiveUI '17, Oldenburg, Germany, September 2017, pp. 254-263

[50] Large, D.R., Burnett, G.E., Antrobus, V., Skrypchuk, L. : 'Driven to Discussion: Engaging drivers in conversation with a digital assistant as a countermeasure to passive taskrelated fatigue', IET Intell. Transp. Sy., 2018, 12, (6), pp. $420-426$

[51] Jacobé de Naurois, C., Bourdin, C., Stratulat, A., et al.: 'Detection and prediction of driver drowsiness using artificial neural network models', Accid. Anal. Prev., 2017, In Press 